\title{
O PAPEL DO ADULTO E DA CRIANÇA COMO PARCEIROS DO DESENVOLVIMENTO EM VYGOTSKY
}

\section{THE ADULT'S AND CHILD'S ROLE AS DEVELOPMENTAL PARTNERS IN VYGOTSKY}

Eulina da Rocha Lordelo ${ }^{1}$

LORDELO, E. R. O Papel do Adulto e da Cnança Como Parceiros do Desenvolvimento em Vygotsky. Rev. Bras. Cresc. Desenv. Hum., São Paulo, 8(1/2), 1998.

Resumo: Este trabalho apresenta uma discussão sobre o papel do adulto e da criança como parceiros desenvolvimentais, à luz da perspectiva vygotskiana e de estudos empíricos que tratam das parcerias adulto-criança e criança-criança e dos seus efeitos para o desenvolvimento. São apresentados alguns resultados de investigações identificando um papel privilegiado do adulto no desenvolvimento da criança, ao lado de outros trabalhos que apontam a alta prioridade motivacional da outra criança como parceiro da brincadeira, bem como efeitos positivos do contato entre crianças no desempenho cognitivo e social. Argumenta-se contra posições mutuamente excludentes, apresentando a literatura que confirma efeitos diferentes e, talvez, complementares, dos dois tipos de experiência.

Palavras-chave: Vygostky; interação adulto-criança; interação criança-criança; parcerias desenvolvimentais.

Uma das mais importantes contribuições de VYGOTSKY à Psicologia e à Educação, a colocação do outro social como pilar da construção do sujeito, tem gerado, até o presente, uma interessante controvérsia, relacionada com o papel do adulto e de outra criança como parceiros do desenvolvimento, particularmente nas implicações práticas do conceito de Zona de Desenvolvimento Proximal (ZPD).

A ZPD é definida como:

“... a distancia entre o nível de desenvolvimento real, que se costuma determinar através da solução independente de problemas, e o nível de desenvolvimento potencial, determinado através da solução de problemas sob a orientação de um adulto ou em colaboração com companheiros mais capazes”. (VYGOTSKY, 1991, p. 97).

A apropriação desse conceito pela psicologia do desenvolvimento atual é marcada por ambiguidades na sua interpretação; de lado, uma tradição de pesquisa que prioriza a assimetria entre os parceiros como uma condição para o desenvolvimento, o que implica em privilegiar uma visão de desenvolvimento guiado por um adulto ou, pelo menos, um parceiro mais velho e competente. De outro lado, um campo de investigação em que o papel da outra criança, evidente nos contextos de criação moderna, em creches e escolas, passa para o plano principal.

1 Doutora em Psicologia pela Universidade de São Paulo. Departamento de Psicologia Faculdade de Educação, Universidade Federal da Bahia. Apoio Capes. End.: Rua Guadalajara, 15, apto. 101, CEP: 40140-460 - Morro do Gato, Salvador, Bahia. Tcl: (071) 245 9375, e-mail: eulina@ufba.br 


\section{O LUGAR DO ADULTO COMO PARCEIRO DO DESENVOLVIMENTO}

Nesse primeiro campo, é postulado um papel diretor do adulto em apoiar e estimular o desenvolvimento da criança nas áreas cognitiva, social e emocional. Entre os mais importantes dados produzidos nessa área estão os resultados de pesquisas que revelam a importância da responsividade do ambiente social para o desenvolvimento inicial do bebê. Um exemplo dessas concepções pode ser visto no trabalho de PAPOUSEK \& PAPOUSEK (1984), que sustentam a necessidade de uma consistente estruturação do ambiente social, na forma de ajuste do comportamento do adulto aos estados comportamentais do bebê, como uma condição para que as trocas iniciais dos bebês com o meio conduzam a integrações bem sucedidas.

Estes autores consideram que o sistema educativo natural ideal representado na interação pais-filhos requer a forma de trocas diádicas apenas um filho e um pai de cada vez, tendo em vista os requerimentos de estabelecer um ambiente social responsivo, em que as trocas comunicativas sejam facilitados pela sincronia entre as ações dos parceiros. Tal sincronia seria possível apenas num ambiente assimétrico, em que o adulto ajusta o seu comportamento ao da criança, esforçando-se ativamente, embora não conscientemente, para ampliar a competência da criança, a partir do seu repertório real. Variações nesse ambiente inicial estariam relacionadas a diferenças no desenvolvimento cognitivo da criança.

Vários estudos corroboram essa hipótese geral, como os que indicam uma relação entre a personalidade materna e os estilos iniciais de interação mãe-filho, responsáveis pela qualidade do vínculo mãe-filho e, mais tarde, por um melhor desenvolvimento emocional, cognitivo e social (WEININGER, 1983; BOCCIA \& CAMPOS, 1989; PEDERSON, MORAN, SITKO, CAMPBELL, GHESQUIRE \& ACTON, 1990; e ISABELLA \& BELSKY, 1991).

De outro lado, pesquisadores como SCHAFFER (1992) acreditam que o adulto (muito mais que a outra criança) tem um papel privilegiado no apoio e suporte aos esforços da criança para compreender e dominar o seu mundo. Grande parte desse papel seria exercido nos chamados Episódios de Envolvimento Conjunto (EEC).

Os EECs são concebidos como situações de interação social em que o adulto esforça-se para ampliar o repertório comportamental da criança, ajudando-a a dominar algum problema novo e progredir para um nível mais alto de competência. Na situação típica de um EEC, a criança, en- quanto está com o interesse focalizado em algum objeto ou acontecimento, pode buscar a mãe quando encontra um aspecto qualquer interessante ou uma dificuldade. Geralmente, em contextos comuns de realização de tarefas domésticas, a mãe responde imediatamente e, após uma breve pausa em que ela localiza o tópico de interesse ou o contexto, fornece o necessário: ajuda, apoio, informação, idéias adicionais, ou simplesmente compartilha o interesse da criança.

SCHAFFER (1992) defende que os EECs desempenham um importante papel no desenvolvimento da criança, tendo em vista o aumento de complexidade do comportamento da criança nessas situações e a hipótese de que quanto mais a criança experimenta essas situações mais seu desenvolvimento cognitivo posterior será acelerado. Essas hipóteses são compatíveis, na interpretação de Schaffer, com a teoria de Vygotsky sobre a importância da troca social, especialmente em situações de assimetria entre os parceiros.

O status das hipóteses formuladas por Schaffer é ainda incerto. Alguns estudos sobre a questão forneceram alguma evidência sobre o papel do adulto em melhorar o desempenho da criança em provas de solução de problemas, confirmando-o (SLADE, 1987; ROCISSANO, SLADE \& LYNCH, 1987; FREUND, 1990), mas outros pesquisadores não obtiveram a mesma relação (KONTOS \& NICHOLAS, 1986).

PEDROSA \& CARVALHO (1995) encontraram brincadeira compartilhada em crianças de menos de dois anos, sem sugestão ou interferência de adultos. As autoras demonstram que as crianças, a partir de elementos da situação (comportamento do outro, objetos), constróem a brincadeira ativamente. Sugerem que a criança recorta uma certa configuração arnbiental e a propõe à outra criança como brincadeira.

OLIVEIRA \& ROSSETTI-FERREIRA (1993) acreditam que as relações entre crianças, propiciam experiências de construção, negociação e articulação de papéis, o que identifica-as como zonas de desenvolvimento proximal.

\section{CONFRONTANDO AS DIFERENTES PERSPECTI VAS}

Assim, diferentes tradições de estudos sobre parceiros de desenvolvimento da criança enfatizam, às vezes em oposição, tanto o adulto como a criança. A confrontação dos resultados, às vezes conflitantes, desses estudos com as concepções de Vygotsky sobre o problema, entretanto, mostra que parte da controvérsia pode ser melhor compreendida na transferência das defi- 
nições gerais para campos mais particulares. Por exemplo, SCHAFFER (1992), embora privilegie o adulto, admite crianças mais velhas como parceiros em EECs. Ele acredita que a natureza do parceiro requerido para gerar mudança envolve diferença de idade; crianças jovens requereriam parceiros de maior competência, enquanto para crianças mais velhas, um parceiro de idade igual ou mais novo será suficiente.

Também a controvérsia sobre o egocentrismo infantil da criança pré-operacional, incluindo a contribuição de VYGOTSKY (1993), após despertar muito debate, vem sendo razoavelmente absorvida pela pesquisa contemporânea, à medida em que os conceitos foram sendo mais esclarecidos e refinados (PIAGET, 1990). No plano empírico, por exemplo, MUSATTI (1986) assinala a tendência recente para integrar desenvolvimento social e cognitivo. Examinando o ponto de vista de Piaget, a autora sustenta que o conceito piagetiano de egocentrismo da criança préoperacional não é incompatível com a intensa sociabilidade da criança nessa fase. A criança egocêntrica é ainda uma criança “pré-social”: “precisamente no sentido em que ela é incapaz de dominar habilidades cognitivas na interação com outras, isto é, de socializar suas operações. Inversamente, em outro sentido, a criança pré-egocêntrica é uma criança profundamente social, não fazendo distinções básicas entre processos cognitivos sociais e individuais”. (MUSATTI, 1986, p. 30).

Musatti acredita, com base na revisão dos estudos na área, que os processos cognitivos subjacentes às interações com pares são os mesmos que operam no comportamento dirigido a objetos. No entanto, variáveis da experiência com pares, como familiaridade, contexto, tamanho do grupo, parecem relevantes em determinar a heterogeneidade do comportamento de crianças do mesmo nível cognitivo.

Musatti sugere que a dimensão egocêntrica do pensamento é um fenómeno funcional, uma vez que aparece em graus diferentes no processo de socialização entre adulto e criança, e entre pares. Assinala a diferença que existe entre os processos de socialização envolvendo os dois tipos de parceiro, uma diferença que é refletida tanto na quantidade e qualidade do egocentrismo como no conjunto do desenvolvimento social.

Ainda segundo MUSATTI (1986), na relação entre adulto e criança, o poder e o conhecimento são muito desiguais. Nessa situação, a criança experimenta maior dificuldade em reconhecer as diferenças entre sua própria atividade cognitiva e a do outro. Elas são menos estimuladas a clarificar seus pontos de vista, comparan- do-os com os do outro. Ao contrário, entre crianças, é mais provável haver verificação e comparação entre pontos de vista e, portanto, cooperação. O parceiro de idade estimula a socialização do conhecimento porque o domínio da consciência sobre os mecanismos que operam na cooperação interpessoal é também estimulado. Assim, a qualidade da relação entre crianças afetaria a dimensão atual e a duração do egocentrismo.

Mas a melhor pista para a compreensão do conflito criança versus adulto como parceiro do desenvolvimento vem, indiretamente, do próprio Vygotsky, quando examina o papel do brinquedo na geração de uma ZPD, como pode ser visto no trecho abaixo:

"Assim, o brinquedo cria uma zona de desenvolvimento proximal da criança. No brinquedo, a criança sempre se comporta além do comportamento habitual de sua idade, além de seu comportamento diário; no brinquedo é como se ela fosse maior do que é na realidade. Como no foco de uma lente de aumento, o brinquedo contém todas as tendências do desenvolvimento sob forma condensada, sendo, ele mesmo, uma grande fonte de desenvolvimento." (VYGOTSKY, 1991, p. 117).

O trecho acima é uma evidência da amplitude do conceito de Zona de Desenvolvimento Proximal que, embora elaborado num contexto educacional, retém poder explanatório para o desenvolvimento como um todo. Não é claro o papel da outra criança nesse quadro, mas ele não é absolutamente incompatível com a idéia de promoção de um contexto desafiador que exige da criança o recurso em níveis mais altos de desempenho.

De falo, um exame do conceito de ZPD nos contextos em que foi aplicado sugere que ele não está realmente vinculado essencialmente a um tipo de parceiro específico. Não obstante o seu uso para enfatizar a natureza assimétrica do contexto apropriado para promover o desenvolvimento, essa vinculação, em Vygotsky, é um artefato das condições empregadas para aplicar o conceito, ou seja, o contexto educacional. Quando considerando os jogos simbólicos característicos do brinquedo de faz-de-conta em crianças pré-escolares, Vygotsky apontou o potencial de desenvolvimento possibilitado pela situação, como salientado por VASCONCELLOS VALSINER ( 1995); não é próprio do conceito ao que parece, a restrição a parceiros de igual competência.

Como assinalado por VALSINE, (1987), o conceito de ZPD, embora retenha, grande poder explanatório, não pode ser verificado empiricamente. Mas as hipóteses geradas pelo conceito são verificáveis. Nesse campo, existem, de fato, 
hipóteses que se põem em conflito, como por exemplo, nos trabalhos de CAMAIONI (1980) e SCHAFFER (1992).

Esse conflito, por sua vez, ainda não está solucionado, em parte pela insuficiência de dados empíricos que sustentem uma ou outra hipótese. Uma outra parte, entretanto, pode ser debitada à conta da formulação do problema, em termos basicamente excludentes, como, por exemplo, nas demonstrações de que o desempenho da criança em companhia de adultos é superior ao observado na companhia de parceiros de igual idade ou vice-versa.

De fato, há boas razões para acreditar que tanto os adultos quanto os pares de idade são parceiros essenciais no desenvolvimento da criança, ainda que não se saiba com certeza os limites em que um dos parceiros diminui seu papel em detrimento do outro. Os estudos que atestam a superioridade do desempenho da criança ora com o adulto, ora com outra criança, não têm que ser visto como imcompatíveis, tanto em função das diferentes dimensões em que podem ser feitas as comparações, como também em função de focalizarem um aspecto recortado do comportamento total da criança.

Em outras palavras, não sabemos como um melhor desempenho de uma criança em urna tarefa, quando acompanhada por um adulto, poderá repercutir em outras áreas do desenvolvimento individual, por exemplo, motivação, auto conceito, auto-estima, entre outros aspectos; mas sabemos, muitas vezes, como experiências do presente relacionam-se com a história passada e repercutem no desenvolvimento futuro do indivíduo, entrelaçando-se com as múltiplas variaveis do seu ambiente. Falar em parceiro privilegiado do desenvolvimento, de todo modo, pode ter significados complexos, impossibilitando sua compreensão em dicotomias do tipo "tudo ou nada" ou "quanto mais, melhor".

Essa posição é encontrada em ROGOFF (1990), quando sugere que diferentes tipos de aprendizagem são favorecidos por diferentes tipos de parceiros. Nesse caso, seria inadequada a comparação, em geral, de pares e adultos. Embora acreditando que a idéia de assimetria dos parceiros seja necessária à teoria de Vygotsky, a autora enfatiza o valor possivelmente curvilinear da sensibilidade da guia do adulto, ou seja, mais sensibilidade não é necessariamente melhor. Esse ponto é ilustrado com exemplos de efeitos perturbadores da exposição a extremos do suporte adulto, que podem variar desde um valor zero, em casos raros que impedem o desenvolvimento de habilidades da espécie (por exemplo, crianças criadas em isolamento social), até o outro extremo de profunda simbiose encontrada ocasionalmente em padrões de interação paisfilho, em que a criança não reconhece o limite entre o seu próprio comportamento e o do outro.

Assim, as hipóteses de que adulto e cnança exercem papéis essencialmente diferentes como parceiros do desenvolvimento não deve implicar, necessariamente, em que um desses papéis deva excluir o outro. Em vez disso, põese em relevo a necessidade de direcionamento da pesquisa para esclarecer como o adulto e a outra criança participam como co-construtores do desenvolvimento, estando incluídas nesse como a importância relativa que cada parceiro detém, em que áreas um parceiro assume maior relevância do que o outro, quando ocorrem as mudanças no equilíbrio entre os parceiros e como os dois sistemas interagem.

\begin{abstract}
This paper discusses the role of the adult and the child as developmental partners, under the Vygotskian view and the empirical studies that deal with adult-child and childchild partnerships and their effects on development. The paper presents some results from other works that identified a privileged role of the adult on child development, and also from studies that point to the high emotional priority of the other child as a playing partner. It also presents results suggesting the positive effects of the contact between children on their cognitive and social perforrnance. The literature presents mutually exclusive positions that we argued against, based on studies that provide evidences of different, and perhaps complementary effects, from both types of experience.
\end{abstract}

Key-words: Vygostsky; adult-child interaction; child-child interaction; developmental partnerships. 


\section{REFERÊNCIAS BIBLIOGRÁFICAS}

BOCCIA, M.; CAMPOS, J. J. Maternal emotional signals, social referencing, and infant's reaction to strangers. In: EISENBERG, N. (ed.). Empathy and related emotional responses. New Directions for Child Development, 44. San Francisco, Jossey Bass, 1989. p.2549. (série).

BRANCO, A. U. A.; CARVALHO, A. M. A.; PEDROSA, M. I. P. C.; GIL, M. S. A. Fluxo de interações entre crianças numa situação de brinquedo em grupo. Psicologia, 15(1): 1327, 1989.

BRUNER, J. S. Nature and uses of immaturity. In: BRUNER, J.S.; JOLLY, A.; SYLVA, K. (eds.). Playits role in development and evolution. London, Penguin Books, 1976.

CAMAIONI, L. L'interazione tra bambini. Roma, Armando Armando, 1980.

CARVALHO, A. M. A.; BERALDO, K.E.A. Interação criança-criança: o ressurgimento de uma área de pesquisa e suas perspectivas. $\mathrm{Ca}$ dernos de Pesquisa, 71: 55-61, 1989.

CARVAlHO, A. M. A.; CARVALHO, J. E. C. Estratégias de aproximação social em crianças de 2 a 6 anos. Psicologia - USP, 1(2): 117126, 1990.

EIBL-EIBENSFELDT, I. Human etholog . New York, Aldine de Gruyter, 1989.

FREUND, L. S. Maternal regulation of children's problem-solving behavior and its impact on children's performance. Child Development, 61(1): 113-126, 1990.

ISABELLA, R.A.; BELSKY, J. Interactional synchrony and the origins of infant-mother attachment: a replication study. Child Development, 62: 373-384, 1991.

KONTOS, S.; NICHOLAS, J.G. Independent problem solving in the development of metacognition. The Journal of Genetic Psychology, 14(4): 481-495, 1986.

LEWIS, M.; BROOKS, J. Sell; other and fear: infant' reaction to people. In: LEWIS, M.; ROSEMBLUM, L. A. (eds.). The origins of fear. New York, Wiley, 1974.

LEWIS, M.; ROSEMBLUM, L .A. Friendship andpeer relations. New York, Wiley, 1975.

LORDELO, E. d. R. Comportamento de cuidado entre crianças. São Paulo, 1986. [Dissertação de Mestrado - Instituto de Psicologia da Universidade de São Paulo].

MUSATTI, T. Early peer relation: the perspectives of Piaget and Vygotsky. In: MUELLER, E.; COOPER, C. (eds.). Process and outcome in peer relationship. New York Academic Press, 1986. p. 25-54.
OLIVEIRA, Z. M. R.; ROSSETTI-FERRFIRA, M. C. O valor da interação criança-criança em creches no desenvolvimento infantil. Cadernos de Pesquisa, 87: 62-70, 1993.

PAPOUSEK, H.; PAPOUSEK, M. Learning and cognitive in the everyday life of human infants. In: ROSENBLATT, J. S. (ed.). Advances in the study of behavior. New York, Academic Press, 1984. v.14, p.127-159.

PEDERSON, D.; MORAN, G.; SITKO, C.; CAMPBELL, K.; GHESQUIRE, K.; ACTON, H. Maternal sensitivity and the security of infantmother attachment: a q-sort study. Child Development, 61: 1974-1983, 1990.

PEDROSA, M. C. I. P.; CARVALHO, A. M. A. A interação social e a constru ão da brincadeira. Cadernos de Pesquisa, 93: 60-65, 1995.

PIAGET, J. Comentários de Piaget sobre as observações críticas de Vygotsky concernentes a duas obras: "A linguagem e o pensamento da criança” e ‘'O raciocínio da criança”. Aberto, 48: 69-77, 1990.

ROCISSANO, L.; SLADE, A.; LYNCH, V. Dyadic synchrony and toddler compliance. Developmental Psychology, 23(5): 698-704, 1987.

ROGOFF, B. Apprenticeship in thinking: Cognitive developmental in social context. New York, Oxford University Press, 1990.

SCHAFFER, H. R. Joint involvement episodes as contexl for development. In: McGURK, $\mathrm{H}$. (ed.) Childhood social development. contemporar perspectives. Hove, Lawrence Erlbaum Associates, 1992.

SLADE, A. A longitudinal study of maternal involvement and symbolic play during the toddle period. Child Development, 58: 367-375, 1987.

THOMAN, E. Changing views of the being anc becoming of infant. In: THOMAN, E. (ed.). Origins of the infant s social responsiveness. New York Wiley, 1979. p.445-459.

VALSINER, J. Culture and the development of children action: a cultural-historical theory of Developmental Psychology. Chichester, Wiley, 1987.

VASCONCELLOS, V.M.R.; VALSINER, J. A perspec tiva co-construtivista em psicologia e na educação. Porto Alegre, Artes Médicas, 1995.

VYGOTSKY, L. S. A formação social da mente. São Paulo, Martins Fontes,1991.

WGOTSKY, L. S. Pensamento e linguagem. São Pau lo, Martins Fontes, 1993.

WEININGER, O. Play of mothers with babies: some relationships between maternal personality and early attachment and development processes. Psychological Reports, 53: 27-42, 1983. 